\title{
IRREDUCIBLE REPRESENTATIONS OF THE GROUP OF MOVEMENTS OF THE EUCLIDEAN PLANE
}

\author{
JAMES SYMONS \\ (Received 13 February 1971, revised 10 January 1972) \\ Communicated by E. Strzelecki
}

\section{Introduction}

It is well known that a wide range of Special Function Theory can be realized by considering unitary representations of certain topological groups.

In this approach it is very important to determine all irreducible continuous unitary representations of the group in question.

For the group of movements this problem was initiated by Vilenkin [6]. Rather restrictive conditions were imposed in this paper and while he returned to the problem in [7], it was still not solved in full generality (among other things the representation space was assumed separable). The first complete solution appears to have been given by Thoma [5]. Here, the method was to show each irreducible continuous unitary representation equivalent to a particular representation in a space of square integrable functions. The form of the operators was given by the expression,

$$
T_{g} f(\psi)=e^{R r \cos (\psi-\phi)} f(\psi-\alpha), \quad R \text { constant, }
$$

where $g$ is the movement determined by a rotation through angle $\alpha$ and a shift $r e^{i \phi}$.

A similar result has been established by Bingen [1]. In this work a representation is understood in the more general sense of a homomorphism from the group into the space of continuous linear operators on some locally convex topological space. However this cannot be considered a generalization since a differentiability condition is imposed on the operators $T_{g}$.

In [2] Gelfand and Shapiro determine the irreducible continuous unitary representations of the group of rotations by considering the infinitesimal operators. This method is easy and natural and hence posed the question: Can the infinitesimal operators be used to characterize all irreducible representations of the group of movements?

In solving this problem many difficulties were encountered. These had their 
origin in that all irreducible representations (with the exception of a trivial representation) are infinite dimensional, and hence the infinitesimal operators are unbounded. In Sections 3, 4 and 5 these difficulties are met by considering the action of the infinitesimal operators on a particular dense subset, $\mathscr{B}$, which has reasonably pleasant properties. Some of the results of [2] carry over directly - for example, Theorem 4 , but they are proved in a different context, and by different methods.

In Sections 7 and 8 the applications of this theory are considered. In particular the Graf addition theorem is derived.

This research was performed in in 1968 and 1969 while I held a Monash University Post-Graduate Award, and constituted a Master Degree. My supervisor was E. Strzelecki and I take this opportunity to express my gratitude to him.

\section{The group of movements}

The group of Movements, $\mathscr{G}$, is the set of all possible transformations of the plane obtained by a rotation about some fixed point (the origin) and applying a constant shift, together with the group operation of iteration of movements.

If the plane is regarded as the complex plane then we may write for $g \in \mathscr{G}$

$$
g=(\alpha, \theta)
$$

where $\theta$ is the angle through which the plane is rotated and $\alpha$ is the complex number of the shift. If by $g(z)$ we understand the complex number obtained by acting upon $z \in \mathbb{C}$ with movement $g$, then

$$
g(z)=e^{i \theta} z+\alpha .
$$

Usually we shall write $g=(x, y, \theta)$ where $x$ and $y$ are the real and imaginary parts of $\alpha$ respectively.

If $g_{1}=\left(x_{1}, y_{1}, \theta_{1}\right), g_{2}=\left(x_{2}, y_{2}, \theta_{2}\right)$ then the group product formula is easily obtained from (1):

$$
g_{1} g_{2}=\left(x_{1}+x_{2} \cos \theta_{1}-y_{2} \sin \theta_{1}, y_{1}+x_{2} \sin \theta_{1}+y_{2} \cos \theta_{1}, \theta_{1}+\theta_{2}\right) \text {. }
$$

The notation $g=(x, y, \theta)$ indicates the natural way to introduce a topology upon $\mathscr{G}$. We define the topology upon $\mathscr{G}$ as the product topology of $R^{2}$ and the circle $\{\theta: 0 \leqq \theta \leqq 2 \pi\}$ where $\theta=0$ and $\theta=2 \pi$ are identified. This topology is obviously locally compact and Hausdorff.

The last remark implies that there exists a left Haar Integral on $\mathscr{G}$, that is,

$$
\int f(g) d g=\int f\left(g_{0} g\right) d g
$$

for integrable function $f(g), f: \mathscr{G} \rightarrow \mathbb{C}$ and for all $g_{0} \in \mathscr{G}$. Writing $g=(x, y, \theta)$ then $f(g)=f(x, y, \theta)$ and th left Haar integral has the form 


$$
\int_{-\infty}^{\infty} \int_{-\infty}^{\infty} \int_{0}^{2 \pi} f(x, y, \theta) d \theta d x d y
$$

\section{Unitary representations of the group of movements}

A unitary representation of $\mathscr{G}$ is a homomorphism $g \rightarrow T_{g}, g \in \mathscr{G}$, where the operators $T_{g}$ are unitary linear operators on some Hilbert space $\mathscr{H}$. That is for $g_{1}, g_{2} \in \mathscr{G}$

$$
T_{g_{1}} T_{g_{2}}=T_{g_{1} g_{2}}
$$

where $T_{g_{1}}$ and $T_{g_{2}}$ are unitary.

In the following the representations will be assumed to be continuous: that is, $\left\|T_{g} \xi-T_{g_{1}} \xi\right\| \rightarrow 0$ for all $\xi \in \mathscr{H}$ as $g \rightarrow g_{1}$ in the topology of $\mathscr{G}$.

A representation $g \rightarrow T_{g}$ of $\mathscr{G}$ is said to be irreducible if the only closed subspaces of $\mathscr{H}$ which are invariant under all operations $T_{g}, g \in \mathscr{G}$, are $\langle 0\rangle$ and $\mathscr{H}$. That is, if $\mathscr{N}$ is a closed subspace of $\mathscr{H}$ and $T_{g}(\mathscr{N}) \subseteq \mathscr{N}$ for all $g \in \mathscr{G}$, then $\mathscr{N}=\langle 0\rangle$ or $\mathscr{H}$.

\section{The space $\mathscr{B}$}

We define $\Omega$ to be the set of complex-valued functions with the following properties. Let $f \in \Omega$ if:

(1) $f(g)=f(x, y, \theta)$ has continuous partial derivatives of all orders.

(2) if $l=f$ or a partial derivative of $f$ and $g_{1}=(h, 0,0),(0, h, 0)$ or $(0,0, h)$, then $l \in L^{2}(\mathscr{G}) \cap L^{1}(\mathscr{G})$ and as $h \rightarrow 0,1 / h\left[l\left(g_{1}^{-1} g\right)-l(g)\right]$ converges in $L^{1}(\mathscr{G})$ to its pointwise limit (it will be shown later that $\Omega \neq \phi$ ). The following are at once apparent.

LEMMA 1. If $f$ is in $\Omega$ then so are $\partial f / \partial x, \partial f / \partial y$ and $\partial f / \partial \theta$.

LEMMA 2. If $g_{1} \in \mathscr{G}$ and $f(g) \in \Omega$ then $f\left(g_{1}^{-1} g\right) \in \Omega$.

We now define $\mathscr{B}=\left\{\eta \in \mathscr{H} ; \eta=\int f(g) T_{g} \xi d g, f \in \Omega, \xi \in \mathscr{H}\right\}$. (The above integration is of a vector valued function, $f(g) T_{g} \xi$. We use the approach of [4] $\S 6$ Section 19. Since $f(g)$ is measurable and bounded, and $T_{g} \xi$ is continuous, the integral exists.)

LEMMA 3. If $\eta \in \mathscr{B}, \eta=\int f(g) T_{g} \xi d g$ with $f \in \mathbf{\Omega}$ and $\xi \in \mathscr{H}$, then $T_{g_{1}} \eta$ $=\int f\left(g_{1}^{-1} g\right) T_{g} \xi d g$.

Proof.

$$
\begin{aligned}
T_{g_{1}} \eta & =T_{g_{1}} \int f(g) T_{g} \xi d g \\
& =\int f(g) T_{g_{1}} T_{g} \xi d g
\end{aligned}
$$


by [4] $\$ 6$ Section 19 Formula 3. Hence

$$
\begin{aligned}
T_{g_{1}} \eta & =\int f(g) T_{g_{1} g} \xi d g \\
& =\int f\left(g_{1}^{-1} g\right) T_{g} \xi d g, \text { by the invariance property. }
\end{aligned}
$$

Corollary. $T_{g}(\operatorname{Span} \mathscr{B}) \subseteq \operatorname{Span} \mathscr{B}(g \in \mathscr{G})$ where Span $\mathscr{B}$ is the linear hull of $\mathscr{B}$.

Proof. By Lemma 2.

\section{The infinitesimal operators}

For $\eta \in \mathscr{H}$ we define

$$
A_{3} \eta=\lim _{h \rightarrow 0} \frac{1}{h}(T(0,0, h) \eta-\eta)
$$

where $T(0,0, h)$ is the representation image of the movement $g=(0,0, h)$.

Similarly we define

$$
\begin{aligned}
& A_{1} \eta=\lim _{h \rightarrow 0} \frac{1}{h}(T(h, 0,0) \eta-\eta) \\
& A_{2} \eta=\lim _{h \rightarrow 0} \frac{1}{h}(T(0, h, 0) \eta-\eta) .
\end{aligned}
$$

It may happen that for particular $\eta$ these limits do not exist. However, this is not so for $\eta \in \mathscr{B}$, as follows from

THEOREM 1. If $\eta \in \mathscr{B}, \eta=\int f(g) T_{g} \xi d g$ then

$$
\begin{aligned}
& A_{1} \eta=\int \frac{\partial f}{\partial x} T_{g} \xi d g \\
& A_{2} \eta=\int \frac{\partial f}{\partial y} T_{g} \xi d g \\
& A_{3} \eta=\int\left(y \frac{\partial f}{\partial x}-x \frac{\partial f}{\partial y}-\frac{\partial f}{\partial \theta}\right) T_{g} \xi d g .
\end{aligned}
$$

Proof. Put $A_{h}=(1 / h)(T(0,0, h)-I)$. By Lemma 3 and some calculation we show

$$
A_{h} \eta=\int \frac{1}{h}\left[f\left(g_{1}^{-1} g\right)-f(g)\right] T_{\theta} \xi d g
$$

where $\eta=\int f(g) T_{g} \xi d g, f \in \Omega, \xi \in \mathscr{H}$ and $g_{1}=(0,0, h)$. Writing $g=(x, y, \theta)$ we see that 


$$
\begin{aligned}
g_{1}^{-1} g & =(0,0,-h)(x, y, \theta) \\
& =(x \cos h+y \sin h, y \cos h-x \sin h, \theta-h) .
\end{aligned}
$$

For fixed $x, y, \theta$ we define

$$
E(k)=f(x \cos k+y \sin k, y \cos k-x \sin k, \theta-k) .
$$

Then $(1 / h)\left[f\left(g_{1}^{-1} g\right)-f(g)\right]=(1 / h)[E(h)-E(0)]$ which implies

$$
\lim _{h \rightarrow 0} \frac{1}{h}\left[f\left(g_{1}^{-1} g\right)-f(g)\right]=E^{\prime}(0) \text {. }
$$

However,

so that

$$
E^{\prime}(k)=\frac{\partial f}{\partial x}(-x \sin k+y \cos k)+\frac{\partial f}{\partial y}(-x \cos k-y \sin k)-\frac{\partial f}{\partial \theta},
$$

$$
E^{\prime}(0)=y \frac{\partial f}{\partial x}-x \frac{\partial f}{\partial y}-\frac{\partial f}{\partial \theta}
$$

thus

$$
\lim _{h \rightarrow 0} \frac{1}{h}\left[f\left(g_{1}^{-1} g\right)-f(g)\right]=y \frac{\partial f}{\partial x}-x \frac{\partial f}{\partial y}-\frac{\partial f}{\partial \theta}, \text { pointwise. }
$$

Now $f \in \Omega$, so this equality holds in $L^{1}(\mathscr{G})$; and this implies

$$
A_{3} \eta=\int\left(y \frac{\partial f}{\partial x}-x \frac{\partial f}{\partial y}-\frac{\partial f}{\partial \theta}\right) T_{\theta} \xi d g
$$

since the integral is a continuous linear functional on $L^{1}(\mathscr{G})$. The other formulae are proved similarly. Q.E.D.

Corollary. Span $\mathscr{B}$ is invariant under $A_{j}, j=1,2,3$; and if $\eta \in \operatorname{Span} \mathscr{B}$ then $A_{j}^{n} \eta, j=1,2,3$ exist for all $n$ and belong to Span $\mathscr{B}$.

Proof. This is immediate from Lemma 1.

Using the forms of the operators $A_{j}$ calculated above, calculation shows the following:

THEOREM 2.

$$
\begin{aligned}
& {\left[A_{3}, A_{1}\right] \eta=A_{2} \eta} \\
& {\left[A_{2}, A_{3}\right] \eta=A_{1} \eta \quad(\eta \in \operatorname{Span} \mathscr{B})}
\end{aligned}
$$

where $[A, B]=A B-B A$ for operators $A, B$.

THEOREM 3. $\mathscr{B}$ contains an eigenvector of $A_{3}$.

Proof. We consider $g=(r, \alpha, \theta)$ where $r e^{i \alpha}=x+i y$. In this case the left Haar integral becomes $\int_{0}^{2 \pi} \int_{0}^{2 \pi} \int_{0}^{\infty} \cdot r d r d \alpha d \theta$. Using Theorem 1 it is easy to show 


$$
A_{3} \eta=\int-\left(\frac{\partial f}{\partial \theta}+\frac{\partial f}{\partial \alpha}\right) T_{g} \xi d g
$$

If $f \in \Omega, f(g)=e^{i n \theta} e^{i m \alpha} h(r)$, where $m, n$ are integers and $h(r)$ is an arbitrary function of $r$, we have:

$$
A_{3} \eta=-i(n+m) \eta
$$

It remains only to show that there exists such an $f \in \Omega$ with $\int f(g) T_{g} \xi d g \neq 0$. We put $h_{k}(r)=r^{k} \exp \left(-r^{2}-\left(1 / r^{2}\right)\right)$, for $k=0, \pm 1, \pm 2 \cdots$, and it is possible to show $e^{i n \theta} e^{i m \alpha} h_{k}(r) \in \Omega$. Assume $\int e^{i n \theta} e^{i m \alpha} h_{k}(r) T_{g} \xi d g=0$, for all $m, n$, and $k$.

Then

$$
\int e^{i n \theta} e^{i m \alpha} r^{k} e^{-r^{2}} e^{-1 / r^{2}}\left(T_{g} \xi, \psi\right) d g=0,
$$

for all $\psi \in \mathscr{H}$ and for all integers $m, n, k$. We put

$$
l(r)=\int_{0}^{2 \pi} \int_{0}^{2 \pi} e^{i n \theta} e^{i m \alpha} e^{-r^{2} / 2} e^{-1 / r^{2}}\left(T_{g} \xi, \psi\right) d \theta d \alpha
$$

then $l(r) \in L^{2}(0, \infty)$ and we have

$$
\int_{0}^{\infty} r^{k} e^{-r^{2} / 2} l(r) r d r=0 .
$$

However the set $\left\{r^{k} e^{-r^{2} / 2} ; k\right.$ a positive integer $\}$ forms a basis for $L^{2}(0, \infty)$ (see [8], Chapter 1 ) so we must have $l(r)=0$. We have shown:

$$
\int_{0}^{2 \pi} \int_{0}^{2 \pi} e^{i n \theta} e^{i m \alpha} e^{-r^{2} / 2} e^{-1 / r^{2}}\left(T_{g} \xi, \psi\right) d \theta d \alpha=0 .
$$

As above the set $\left\{e^{i n \theta} ; n\right.$ an integer $\}$ forms a basis for $L^{2}(0,2 \pi)$, and we may argue as before. The final result is

$$
\left(T_{g} \xi, \psi\right)=0 \quad(\psi \in \mathscr{H}),
$$

which is impossible unless $\xi=0$.

Q.E.D.

The operators $A_{j} ; j=1,2,3$ have the following property. If $\eta_{1}, \eta_{2} \in \operatorname{Span} \mathscr{B}$, then

$$
\left(A_{j} \eta_{1}, \eta_{2}\right)=\left(\eta_{1},-A_{j} \eta_{2}\right) ; \quad j=1,2,3
$$

Indeed,

$$
\left(\frac{1}{h}(T(0,0, h)-I) \eta_{1}, \eta_{2}\right)=\left(\eta_{1}, \frac{1}{h}(T(0,0,-h)-I) \eta_{2}\right),
$$

since the operators $T_{g}, g \in \mathscr{G}$, are unitary; taking limits, we have the relation. We now define 


$$
i A_{j}=H_{j} ; j=1,2,3,
$$

and the above relation may be written

$$
\left(H_{j} \eta_{1}, \eta_{2}\right)=\left(\eta_{1}, H_{j} \eta_{2}\right) ; \quad j=1,2,3 \text {. }
$$

(If we use the terminology of [4] $\$ 5$ Section 9 we may call the operators $H_{J}$ symmetric.)

It is more convenient to work with certain linear combinations of the $H_{j}$. We define

$$
\begin{aligned}
& H_{+}=H_{1}+i H_{2} \\
& H_{-}=H_{1}-i H_{2} .
\end{aligned}
$$

Direct calculation then establishes the following relations

$$
\begin{aligned}
& {\left[H_{+}, H_{3}\right]=-H_{+}} \\
& {\left[H_{-}, H_{3}\right]=H_{-}} \\
& {\left[H_{+}, H_{-}\right]=0} \\
& \left(H_{+} \eta_{1}, \eta_{2}\right)=\left(\eta_{1}, H_{-} \eta_{2}\right)
\end{aligned}
$$

- for $\eta_{1}, \eta_{2} \in \operatorname{Span} \mathscr{B}$.

THEOREM 4. Let $f_{\lambda} \in \mathscr{B}$ be an eigenvector of $H_{3}$ with eigenvalue $\lambda$. Then if $H_{+} f_{\lambda} \neq 0$ it is an eigenvector of $H_{3}$ with eigenvalue $\lambda+1$. Similarly if $H_{-} f_{\lambda} \neq 0$ then $H_{-} f_{\lambda}$ is an eigenvector of eigenvalue $\lambda-1$.

Proof. $H_{3} H_{+} f_{\lambda}=\left[H_{3}, H_{+}\right] f_{\lambda}+H_{+} H_{3} f_{\lambda}=H_{+} f_{\lambda}+\lambda H_{+} f_{\lambda}=(\lambda+1) H_{+} f_{\lambda}$ where $f_{\lambda} \in \mathscr{B}$ is an eigenvector of eigenvalue $\lambda$. The dual result is proved similarly.

Q.E.D.

We shall prove eventually that if $T_{g}$ is irreducible then $H_{+} H_{-} f_{\lambda}=M f_{\lambda}$, for some $M \in \mathbb{R}$.

LemMA 4. If $g \rightarrow T_{g}$ is irreducible then Span $\mathscr{B}$ is dense in $\mathscr{H}$.

Proof. One considers $\overline{\operatorname{Span} \mathscr{B}}$. This is invariant due to the corollary to Lemma 3.

In general if $A$ is an operator on $\mathscr{H}, \mathscr{D}(A)$ (Domain $A$ ) dense in $\mathscr{H}$, then $A$ is self-adjoint if $A=A^{*}$. The following three results are from $\$ 5$ Section 9 of [4].

Proposition 1. $A^{*}$ is a closed linear operator.

Proposition 2. If $A$ is closed and $\mathscr{D}(A)$ dense in $\mathscr{H}$, then $A^{*} A$ is self adjoint.

Proposition 3. If the linear operator $A$ with dense domain has the closure $\tilde{A}$ then 


$$
A^{* *}=\tilde{A} \supseteq A \text {. }
$$

We shall also need the following.

THEOREM 5. Let $g \rightarrow T_{g}$ be an irreducible representation of $\mathscr{G}$. Let $A$ be an (a priori) unbounded self-adjoint operator such that $\mathscr{D}\left(T_{g} A\right) \subseteq \mathscr{D}\left(A T_{g}\right)$ and for $\eta \in \mathscr{D}\left(T_{g} A\right), T_{g} A \eta=A T_{g} \eta$; then $A=M I$ for some real $M$.

(See [4] \$29 Section 3. The result for rings is established in $\$ 17$, Section 6, Proposition II and the result for groups is a consequence of the relationship established in \$29.)

We define $H=H_{+}^{* *} H_{+}^{*}$ and from Proposition 1 and Proposition 2 we have:

Proposition 4. $H$ is self-adjoint.

Proposition 5. $\mathrm{H} \supseteq H_{+} H_{-}$(i.e. $H$ is an extension of $H_{+} H_{-}$).

PROOF.

$$
\begin{aligned}
H_{+}^{*} & =\left(H_{1}+i H_{2}\right)^{*} \\
& \supseteq H_{1}^{*}-i H_{2}^{*} \\
& \supseteq H_{1}-i H_{2},
\end{aligned}
$$

since the operators $H_{1}$ and $H_{2}$ are symmetric. Since $H_{-}^{*}$ is closed, $H_{+}$admits of a closure, and by our Proposition 3:

$$
H_{+}^{* *} \supseteq H_{+}
$$

Q.E.D.

Proposition 6. If $A$ is self-adjoint then

$$
\mathscr{D}(A)=\left\{\xi \in \mathscr{H} ; \int_{-\infty}^{\infty}|\lambda|^{2} d\|P(\lambda) \xi\|^{2}<\infty\right\}
$$

and

$$
A \xi=\int_{-\infty}^{\infty} \lambda d P(\lambda) \xi \text { for } \xi \in \mathscr{D}(A) .
$$

Note. $P(\lambda)$ is the spectral operator function and this result is the spectral theorem for unbounded linear operators. See [4], \$17.

Proposition 7. If $\xi \in \mathscr{B}$ then $T_{g} H \xi=H T_{g} \xi$.

Proof. These expressions are both well defined, (see the Corollary to Lemma 3 and Proposition 5). To prove the equality we make use of Lemma 3 and the form of the infinitesimal operators calculated in Theorem 1.

Proposition 8. $P(\lambda) T_{g}=T_{g} P(\lambda)$.

Proof. We adapt the proof of Proposition VII $\$ 17$ of [4]. It is straight- 
forward to show from Proposition 7 that $T_{g}$ commutes with $U$ (as defined in [4]) for vectors in $\mathscr{B}$. Since $\mathscr{B}$ is dense in $\mathscr{H}$ and both $T_{g}$ and $U$ are unitary operators this property can be extended to $\mathscr{H}$. The argument then proceeds as in the reference.

Proposition 9. $\mathscr{D}\left(T_{\theta} H\right) \subseteq \mathscr{D}\left(H T_{\theta}\right)$, and for $\xi \in \mathscr{D}\left(T_{q} H\right)$,

$$
T_{g} H \xi=H T_{g} \xi \text {. }
$$

PROOF. This is immediate from Propositions 6 and 8.

We can now apply Theorem 5 to obtain:

THEOREM 6. Let $g \rightarrow T_{g}$ be an irreducible representation of $\mathscr{G}$. Then $H_{+} H_{-} \eta=M \eta(\eta \in \operatorname{Span} \mathscr{B})$ for some real $M$.

\section{Exponential formulae}

In this section we will determine the form of the operators $T_{g}$ in terms of exponentials. The symbol $f_{\lambda}$ will denote a non-zero eigenvector of $H_{3}$ with eigenvalue $\lambda$ where $f_{\lambda} \in \mathscr{B}$. $\mathscr{B}\left(f_{\lambda}\right)$ will denote the set

$$
\left\{f_{\lambda}\right\} \bigcup\left\{H_{+}^{n} f_{\lambda} ; n \text { a positive integer }\right\} \bigcup\left\{H_{-}^{n} f_{\lambda} ; n \text { a positive integer }\right\}
$$

and $\operatorname{Span} \mathscr{B}\left(f_{\lambda}\right)$ will denote the set of all finite linear combinations of the vectors of $\mathscr{B}\left(f_{\lambda}\right)$.

THEOREM 7. Let $g \rightarrow T_{g}$ be a continuous unitary representation of $\mathscr{G}$. If $\eta \in \operatorname{Span} \mathscr{B}$,

$$
T(0,0, \theta) \eta=\sum_{k=0}^{n-1} \frac{\theta^{k}}{k !} A_{3}^{k} \eta+\frac{1}{(n-1) !} \int_{0}^{\theta}(\theta-\tau)^{n-1} T(0,0, \tau) A_{3}^{n} \eta d \tau .
$$

Proof. The reference is [3] Theorem 11.6.3. Assuming the representation to be continuous is a much stronger condition than $(0, A)$ summability.

Corollary. If $\left\|A_{3}^{n} \eta\right\| \leqq K^{n}\|\eta\|$ for some $K \geqq 0$ then we have

$$
T(0,0, \theta) \eta=\sum_{k=0}^{\infty} \frac{\theta^{k}}{k !} A_{3}^{j} \eta \text { for } \eta \in \operatorname{Span} \mathscr{B} ;
$$

and similar results hold for $T(x, 0,0)$ and $T(0, y, 0)$.

Proof. One shows that $\left\|\int_{0}^{\theta}(\theta-\tau)^{n-1} T(0,0, \tau) A_{3}^{n} \eta d \tau\right\| \rightarrow 0$ as $n \rightarrow \infty$.

We can now proceed to the calculation of the form of an irreducible representation of $\mathscr{G}$. We shall denote $\sum_{k=0}^{\infty}\left(\theta^{k} / k !\right) A_{3}^{k} \eta$ by $\exp \left(\theta A_{3}\right) \eta$.

THEOREM 8. Let $g \rightarrow T_{g}$ be irreducible. If $M=0$ then the representation is 1-dimensional of the form $T(x, y, \theta)=e^{i k \theta}$ where $k$ is a constant integer. 
Proof. Assume $M=0$. Let $f_{\lambda}$ be an eigenvector of $H_{3}$ with eigenvalue $\lambda$. $\lambda \in \mathbb{R}$, since $H_{3}$ is self adjoint. Now

$$
\left(H_{-} f_{\lambda}, H_{-} f_{\lambda}\right)=\left(f_{\lambda}, H_{+} H_{-} f_{\lambda}\right)=\left(f_{\lambda}, M f_{\lambda}\right)=\left(f_{\lambda}, 0\right)=0,
$$

so $H_{-} f_{\lambda}=0$. Similarly $H_{+} f_{\lambda}=0$, so in general $H_{+}^{n} f_{\lambda}=H_{-}^{m} f_{\lambda}=0$ for all positive integers $n, m$. Hence $A_{1}^{n} f_{\lambda}=A_{2}^{m} f_{\lambda}=0$ and trivially

$$
\left\|A_{j}^{m} f_{\lambda}\right\| \leqq\left\|f_{\lambda}\right\| ; \quad j=1,2 .
$$

Thus by the corollary to Theorem 7 we have

$$
\begin{aligned}
& T(x, 0,0) f_{\lambda}=\exp \left(x A_{1}\right) f_{\lambda}=f_{\lambda}, \\
& T(0, y, 0) f_{\lambda}=\exp \left(y A_{2}\right) f_{\lambda}=f_{\lambda} .
\end{aligned}
$$

Since $f_{\lambda}$ is an eigenvector of $A_{3}$ we have that $A_{3}^{n} f_{\lambda}=(-i \lambda)^{n} f_{\lambda}, \lambda \in \mathbb{R}$, and this gives $\left\|A_{3}^{n} f_{\lambda}\right\|=|\lambda|^{n}\left\|f_{\lambda}\right\|$. So by the same corollary,

$$
\begin{aligned}
T(0,0, \theta) f_{\lambda} & =\exp \left(\theta A_{3}\right) f_{\lambda} \\
& =f_{\lambda}+\theta A_{3} f_{\lambda}+\frac{1}{2 !}\left(\theta A_{3}\right)^{2} f_{\lambda}+\cdots \\
& =f_{\lambda}+(-i \lambda \theta) f_{\lambda}+\frac{1}{2 !}(-i \lambda \theta)^{2} f_{\lambda}+\cdots \\
& =e^{-i \lambda \theta} f_{\lambda} .
\end{aligned}
$$

Since $T(x, y, \theta)=T(x, 0,0) T(0, y, 0) T(0,0, \theta)$ we have $T_{g} f_{\lambda}=e^{i k \theta} f_{\lambda}$ for some real $k$, and since $T(0,0,2 n \pi)=I$ for integer $n$, we must have $k$ an integer. Clearly this formula holds in the one-dimensional space

$$
\mathbb{C} f_{\lambda} \subseteq \mathscr{H} \text {. }
$$

Hence $\mathbb{C} f_{\lambda}$ is invariant under all operators $T_{g}, g \in \mathscr{G}$, and the irreduciblility condition implies $\mathbb{C} f_{\lambda}=\mathscr{H}$.

LeMMA 5. If $g \rightarrow T_{g}$ is an irreducible representation and $M \neq 0$ then $H_{+}^{n} f_{\lambda} \neq 0$ and $H_{-}^{m} f_{\lambda} \neq 0$ for all positive integers $m, n$.

Proof. If $M \neq 0$, let $n$ be the least positive integer such that

$$
H_{+}^{n} f_{\lambda}=0 .
$$

Then $0=H_{-} H_{+}^{n} f_{\lambda}=H_{-} H_{+} H_{+}^{n-1} f_{\lambda}=M H_{+}^{n-1} f_{\lambda}$, which implies that $H_{+}^{n-1} f_{\lambda}=0$, a contradiction.

In the light of this result we henceforth assume $M \neq 0, H_{+}^{n} f_{\lambda} \neq 0$ and $H_{-}^{n} f_{\lambda} \neq 0$.

THEOREM 9. Let $g \rightarrow T_{g}$ be irreducible. Then $M$ is positive and if $N$ is 
the positive square root of $M$, then $H_{ \pm}^{n} f_{\lambda}=N^{n} f_{\lambda \pm n}$ for integers $n \geqq 0$, where $\left(f_{\lambda \pm n}, f_{\lambda \pm n}\right)=1$.

Proof. We write $H_{+} f_{\lambda}=\alpha_{1} f_{\lambda+1}$. Clearly $f_{\lambda+1}$ may be selected so that $\left(f_{\lambda+1}, f_{\lambda+1}\right)=1$ and $\alpha_{1}>0$. In general we define

$$
H_{+} f_{\lambda+n}=\alpha_{n+1} f_{\lambda+n+1},\left(f_{\lambda+n+1}, f_{\lambda+n+1}\right)=1 \text {, }
$$

where $\alpha_{n+1}>0$. In the same way we define

$$
H_{-} f_{\lambda-n}=\beta_{n+1} f_{\lambda-n-1}
$$

where $\beta_{n+1}>0$ and $\left(f_{\lambda-n-1}, f_{\lambda-n-1}\right)=1$, for all $n$. Since $f_{\lambda \pm n} \in \mathscr{B}$ we have

$$
H_{+} H_{-} f_{\lambda \pm n}=M f_{\lambda \pm n} .
$$

Now $H_{-} f_{\lambda+n+1}=H_{-}\left(1 / \alpha_{n+1}\right) H_{+} f_{\lambda+n}=\left(1 / \alpha_{n+1}\right) H_{-} H_{+} f_{\lambda+n}=\left(M / \alpha_{n+1}\right) f_{\lambda+n}$ and a similar calculation gives

$$
H_{+} f_{\lambda-n-1}=\frac{M}{\beta_{n+1}} f_{\lambda-n} .
$$

Since $\left(H_{+} \eta_{1}, \eta_{2}\right)=\left(\eta_{1}, H_{-} \eta_{2}\right)$ for $\eta_{1}, \eta_{2} \in$ Span $\mathscr{B}$, it follows that

and

$$
\left(H_{+} f_{\lambda+n}, f_{\lambda+n+1}\right)=\left(\alpha_{n+1} f_{\lambda+n+1}, f_{\lambda+n+1}\right)=\alpha_{n+1}
$$

$$
\left(H_{+} f_{\lambda+n}, f_{\lambda+n+1}\right)=\left(f_{+n}, H_{-} f_{\lambda+n+1}\right)=\left(f_{\lambda+n}, \frac{M}{\alpha_{n+1}} f_{\lambda+n}\right)=\frac{M}{\alpha_{n+1}} .
$$

Hence $\left(M / \alpha_{n+1}\right)=\alpha_{n+1}$, that is, $M=\alpha_{n+1}^{2}>0$. By considering $\left(H_{-} f_{\lambda-n}, f_{\lambda-n-1}\right)$ we obtain the relation

$$
\beta_{n+1}^{2}=M \text {. }
$$

We put $+\sqrt{M}=N$ and we have

as required.

$$
\beta_{n+1}=\alpha_{n+1}=N \text {, }
$$

THEOREM 10. If $g \rightarrow T_{g}$ is irreducible and $\eta \in \operatorname{Span} \mathscr{R}\left(f_{\lambda}\right)$, then

$$
T(x, y, \theta) \eta=\exp \left(x A_{1}+y A_{2}\right) \exp \left(\theta A_{3}\right) \eta \text {. }
$$

Proof. Let $f_{\lambda+n} \in \mathscr{B}\left(f_{\lambda}\right)$. We have $A_{1}=(1 / 2 i)\left(H_{+}+H_{-}\right)$. Hence, since $H_{+}$ and $H_{-}$commute:

$$
A_{1}^{m} f_{\lambda+n}=\left(\frac{1}{2 i}\right)^{m}\left[H_{+}^{m}+\left(\begin{array}{c}
m \\
1
\end{array}\right) H_{+}^{m-1} H_{-}+\cdots+\left(\begin{array}{c}
m \\
m-1
\end{array}\right) H_{+}^{1} H_{-}^{m-1}+H_{-}^{m}\right] f_{\lambda+n},
$$

and calculation shows

$$
\left\|A_{1}^{m} f_{\lambda+n}\right\| \leqq\left(\frac{N}{2}\right)^{m}\left[1+\left(\begin{array}{c}
m \\
1
\end{array}\right)+\cdots+\left(\begin{array}{c}
m \\
m-1
\end{array}\right)+1\right]=\left(\frac{N}{2}\right)^{m} \cdot 2^{m}=N^{m} .
$$


The dual result for $A_{2}$ is proved similarly; thus

$$
\left\|A_{i}^{m} f_{\lambda+n}\right\| \leqq N^{m} \quad i=1,2 .
$$

Also, since $f_{\lambda+n}$ is an eigenvector of $A_{3}$ we have

$$
\left\|A_{3}^{m} f_{\lambda+n}\right\|=|\lambda+n|^{m} \text {. }
$$

Hence by the corollary to Theorem 7 we have

$$
\begin{aligned}
& T(0,0, \theta) f_{\lambda+n}=\exp \left(\theta A_{3}\right) f_{\lambda+n} \\
& T(x, 0,0) f_{\lambda+n}=\exp \left(x A_{1}\right) f_{\lambda+n} \\
& T(0, y, 0) f_{\lambda+n}=\exp \left(y A_{2}\right) f_{\lambda+n}
\end{aligned}
$$

So

$$
\begin{aligned}
T(x, y, \theta) f_{\lambda+n} & =T(x, 0,0) T(0, y, 0) T(0,0, \theta) f_{\lambda+n} \\
& =T(x, 0,0) T(0, y, 0) \exp \left(\theta A_{3}\right) f_{\lambda+n} .
\end{aligned}
$$

Now $T(0,0, \theta) f_{\lambda+n} \in \mathscr{B}$ (Lemma 3). Moreover $H_{3}$ commutes with $T(0,0, \theta)$. So

$$
H_{3} T(0,0, \theta) f_{\lambda+n}=T(0,0, \theta) H_{3} f_{\lambda+n}=(\lambda+n) T(0,0, \theta) f_{\lambda+n} .
$$

Thus $f_{\lambda+n}^{\prime}=T(0,0, \theta) f_{\lambda+n}$ is a non-zero eigenvector of $H_{3}$. Starting with $f_{\lambda+n}^{\prime}$ as $f_{\lambda}$ we may reproduce the results of Theorem 9 and in particular obtain formulae (11). Hence

$$
T(0, y, 0) f_{\lambda+n}^{\prime}=\exp \left(y A_{2}\right) f_{\lambda+n}^{\prime} .
$$

Moreover $A_{1} A_{2}=A_{2} A_{1}$, so $A_{1} \exp \left(y A_{2}\right)=\exp \left(y A_{2}\right) A_{1}$, and this implies

$$
\begin{aligned}
A_{1}^{m} T(0, y, 0) f_{\lambda+n}^{\prime} & =\exp \left(y A_{2}\right) A_{1}^{m} f_{\lambda+n}^{\prime} \\
& =\exp \left(y A_{2}\right)\left(\frac{1}{2 i}\right)^{m}\left(H_{+}+H_{-}\right)^{m} f_{\lambda+n}^{\prime} .
\end{aligned}
$$

$(1 / 2 i)^{m}\left(H_{+}+H_{-}\right)^{m} f_{\lambda+n}^{\prime}$ is a linear combination of eigenvectors of $H_{3}$. Clearly formulae (11) apply to such vectors and the expression on the right is just:

$$
T(0, y, 0)\left(\frac{1}{2 i}\right)^{m}\left(H_{+}+H_{-}\right)^{m} f_{\lambda+n}^{\prime} .
$$

The unitariness of $T(0, y, 0)$ and a similar calculation to that which derived (10) give

$$
\left\|A_{1}^{m} T(0, y, 0) f_{\lambda+n}^{\prime}\right\| \leqq N_{1}^{m}
$$

where $N_{1}$ is the $N$ of Theorem 9 for $f_{\lambda+n}^{\prime}=f_{\lambda}$. Hence by the corollary to Theorem 7 we have

$$
T(x, 0,0) T(0, y, 0) f_{\lambda+n}^{\prime}=\left(\exp x A_{1} \exp y A_{2} \exp \theta A_{3}\right) f_{\lambda+n} .
$$

So 


$$
T(x, y, \theta) f_{\lambda+n}=\left(\exp x A_{1} \exp y A_{2} \exp \theta A_{3}\right) f_{\lambda+n}
$$

- and since $A_{1} A_{2}=A_{2} A_{1}$ it is easy to verify that

$$
\exp x A_{1} \exp y A_{2}=\exp \left(x A_{1}+y A_{2}\right) \text {. }
$$

The generalization from $\mathscr{B}\left(f_{\lambda}\right)$ to $\operatorname{Span} \mathscr{B}\left(f_{\lambda}\right)$ is now immediate.

THEOREM 11. Let $g \rightarrow T_{g}$ be an irreducible unitary representation of $\mathscr{G}$. Then $\mathscr{H}=\overline{\operatorname{Span} \mathscr{B}\left(f_{\lambda}\right)}$.

Proof. Span $\mathscr{B}\left(f_{\lambda}\right)$ is invariant under all operators $H_{+}, H_{-}, H_{3}$. Using the form of the operators on $\operatorname{Span} \mathscr{B}\left(f_{\lambda}\right)$ calculated in Theorem 9 we have the result.

COROLlaRY. Any irreducible representation of $\mathscr{G}$ is separable.

Proof. In fact the countable basis of $\mathscr{H}$ is $\mathscr{B}\left(f_{\lambda}\right)$.

Finally we note that

$$
T(0,0, \theta) f_{\lambda+n}=\exp \theta A_{3} f_{\lambda+n}=e^{-i(\lambda+n) \theta} f_{\lambda+n} .
$$

Since $T(0,0,2 m \pi)=I$ for integer $m$ it follows that $\lambda$ is an integer. By Theorem 4 we may take $\lambda=0$. Thus we have established the following:

THEOREM. Let $g \rightarrow T_{g}$ be an irreducible continuous unitary representation of the group of movements in the Space $\mathscr{H}$, dimension $\mathscr{H} \neq 1$. Then $\mathscr{H}$ is separable (and infinite dimensional) and there exists an orthonormal basis of $\mathscr{H}, \mathscr{B}(f)=\left\{f_{n} ; n\right.$ an integer $\}$ upon which the infinitesimal operators have the form

$$
\begin{aligned}
& A_{1} f_{n}=-\frac{i}{2} N f_{n+1}-\frac{i}{2} N f_{n-1} \\
& A_{2} f_{n}=-\frac{1}{2} N f_{n+1}+\frac{1}{2} N f_{n-1} \\
& A_{3} f_{n}=-i n f_{n},
\end{aligned}
$$

where $N>0$. Moreover if $\eta \in \operatorname{Span} \mathscr{B}(f)$,

$$
T_{g} \eta=\exp \left(x A_{1}+y A_{2}\right) \exp \theta A_{3} \eta
$$

where $g=(x, y, \theta)$.

\section{Infinite matrices}

We have shown that for any irreducible representation, $\mathscr{H}$ is separable. Thus we may regard $\mathscr{H}$ as the set of column matrices of the form 


$$
\eta=\left[\begin{array}{c}
\cdot \\
\cdot \\
\cdot \\
\eta_{-1} \\
\eta_{0} \\
\eta_{1} \\
\cdot \\
\cdot \\
\cdot
\end{array}\right] \text { where formerly we wrote } \eta=\sum_{-\infty=n}^{\infty} \eta_{n} f_{n}
$$

(where $\left.\sum_{-\infty=n}^{\infty}\left|\eta_{n}\right|^{2}<\infty\right)$. Moreover on this space any linear operator may be represented as an infinite matrix: $T=\left(t_{m n}\right)$ where $T \eta$ is the column matrix with $\sum_{-\infty=k}^{\infty} t_{m k} \eta_{k}$ in the $m$ th place. In this notation $f_{n}$ is just the column matrix with 1 in the $n$th place and zeros elsewhere. It is easily seen that the infinitesimal operators have the forms

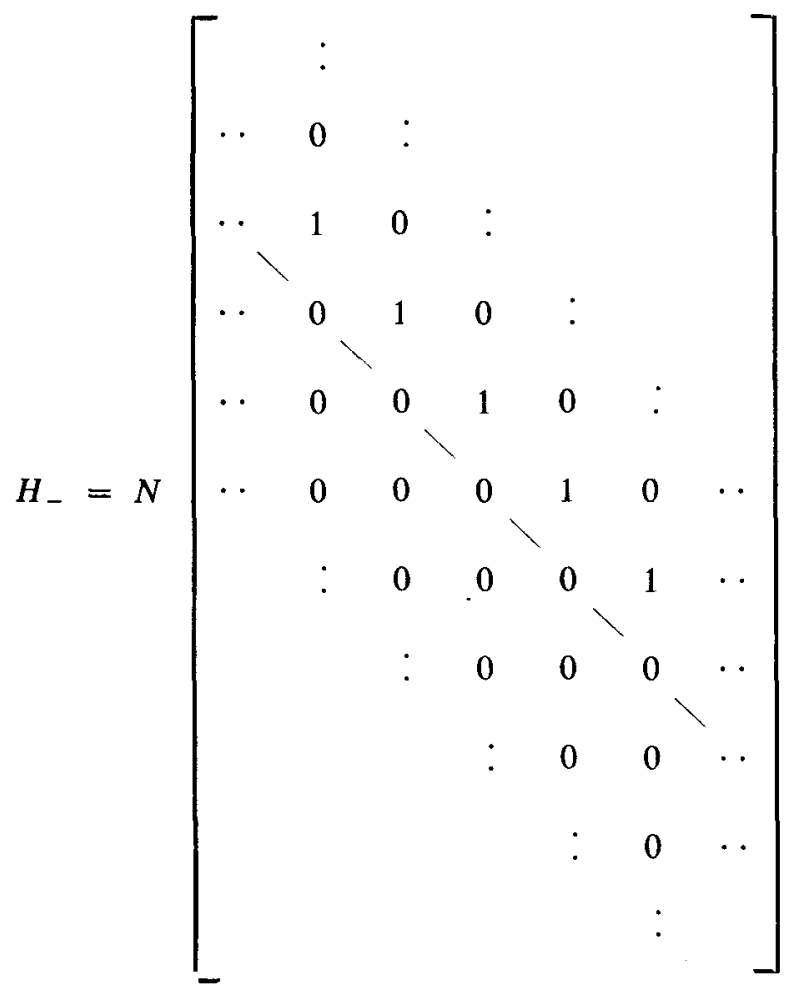




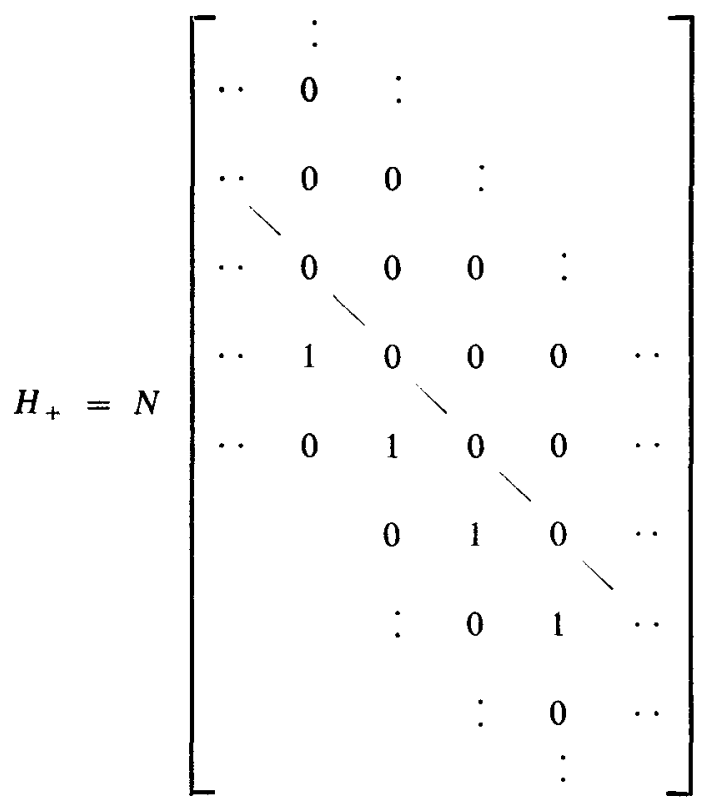

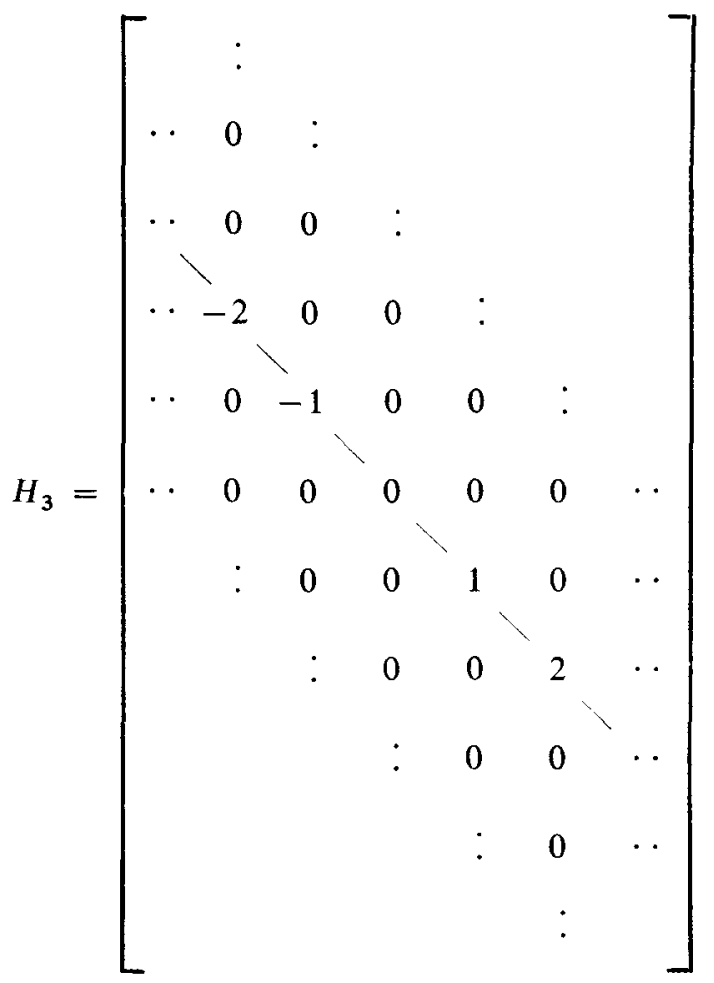


- that is the $m n$th entries of $H_{+}, H_{-}$and $H_{3}$ are $N \delta_{m, n+1}, N \delta_{m+1, n}$ and $m \delta_{m, n}$ respectively.

Using these expressions for $H_{+}, H_{-}, H_{3}$ it is possible, though tedious, to prove the following converse of the theorem of Section 5 .

THEOREM. Let $\mathscr{H}$ be the space of infinite column matrices,

$$
\eta=\left[\begin{array}{c}
\cdot \\
\cdot \\
\eta_{-1} \\
\eta_{0} \\
\eta_{1} \\
\cdot \\
\cdot
\end{array}\right] \text { where } \sum_{-\infty=n}^{\infty}\left|\eta_{n}\right|^{2}<\infty \text { and let the matrices }
$$

$H_{+}, H_{-}, H_{3}$ be defined as above. Put $f_{n}$ as the column matrix with 1 in the $n$th place. Then $\operatorname{Span} \mathscr{B}(f)$, the set of column matrices with only finite non-zero entries, is dense in $\mathscr{H}$. Moreover for $\eta \in \operatorname{Span} \mathscr{B}(f)$ the matrix

$$
T=\exp \frac{x}{2 i}\left(H_{+}+H_{-}\right) \exp -\frac{y}{2}\left(H_{+}-H_{-}\right) \exp \left(-i \theta H_{3}\right) \eta
$$

is defined for $x, y, \theta \in \mathbb{R}$. Writing $T_{g}$ (where $g=(x, y, \theta)$ ) for the unique extension to $\mathscr{H}$ of this operator $T$ we have that $g \rightarrow T_{g}$ is an irreducible continuous unitary representation of $\mathscr{G}$.

\section{The matrix elements of an irreducible representation}

It has been shown (for a dense subset of $\mathscr{H}$ ) that

$$
\begin{aligned}
T_{g} & =\exp x A_{1} \exp y A_{2} \exp \theta A_{3} \\
& =\exp \frac{x}{2 i}\left(H_{+}+H_{-}\right) \exp \frac{y}{2}\left(H_{-}-H_{+}\right) \exp \frac{\theta}{i} H_{3} \\
& =\exp \frac{x}{2 i} H_{+} \exp \frac{x}{2 i} H_{-} \exp \frac{y}{2} H_{-} \exp \left(-\frac{y}{2} H_{+}\right) \exp \frac{\theta}{i} H_{3}
\end{aligned}
$$

and the matrices $H_{+}, H_{-}, H_{3}$ have the forms $N\left(\delta_{m, n+1}\right), N\left(\delta_{m+1, n}\right),\left(n \delta_{m, n}\right)$ respectively. (By $\left(\delta_{m, n+1}\right)$ etc. we understand the matrix with $\delta_{m, n+1}$ in the $m, n$th 
position.) The powers of $H_{+}, H_{-}$are easy to calculate. In fact

$$
\begin{aligned}
& H_{+}^{p}=N^{p}\left(\delta_{m-p, n}\right) \\
& H_{-}^{p}=N^{p}\left(\delta_{m+p, n}\right)
\end{aligned}
$$

and from this it follows that

$$
\begin{aligned}
& \exp \frac{x}{2 i} H_{+}=\sum_{d=0}^{\infty}(-i)^{k} \frac{1}{k !}\left(\frac{N x}{2}\right)^{k}\left(\delta_{m+k, n}\right) \text { and } \\
& \exp \frac{x}{2 i} H_{-}=\sum_{k=0}^{\infty}(-i)^{k} \frac{1}{k !}\left(\frac{N x}{2}\right)^{k}\left(\delta_{m, n+k}\right) .
\end{aligned}
$$

By multiplying these two matrices and some calculation it can be shown that the $m, n$th entry of $\exp x A_{1}$ is

$$
(-i)^{m-n} J_{m-n}(N x)
$$

where $J_{m-n}(x)$ is the Bessel function of order $m-n$, and a similar approach shows that the $m, n$th entry of $\exp y A_{2}$ is $J_{m-n}(N y)$.

Using the fact that $H_{3}$ is a diagonal matrix it is easy to show $\exp \theta A_{3}=$ $\left(e^{-i n \theta} \delta_{m, n}\right)$, so that the final result is that the $m, n$th entry of $T_{g}=\exp \left(x A_{1}+y A_{2}\right)$ $\exp \theta A_{3}$ is:

$$
\sum_{-\infty=k}^{\infty}(-i)^{m-k} J_{m-k}(N x) J_{k-n}(N y) e^{-i n \theta}
$$

\section{Some relations between the Bessel functions}

The fundamental relation

$$
T_{g_{1}} T_{g_{2}}=T_{g_{1} g_{2}},
$$

satisfied by the operators $T_{g}$ is, in effect, an addition formula for Bessel functions As a preliminary we put

$$
g_{1}=(0,0, \theta), g_{2}=(0, y, 0)
$$

so that

$$
g_{1} g_{2}=(y \cos \theta, y \sin \theta, \theta) .
$$

Substituting in (13) from (12) we have

$$
\begin{gathered}
\sum_{-\infty=k}^{\infty}(-i)^{m-k} J_{m-k}(N y \cos \theta) J_{k-n}(N y \sin \theta) e^{-i n \theta} \\
=(-i)^{m-n} J_{m-n}(N y) e^{-i m \theta}
\end{gathered}
$$


so that

$$
J_{m-n}(y)=e^{i(m-n)(\theta+\pi / 2)} \sum_{-\infty=k}^{\infty}(-i)^{m-k} J_{m-k}(y \cos \theta) J_{k-n}(y \sin \theta)
$$

- which expresses a Bessel function in terms of its "components". This formula enables us to simplify the general element of $T_{g}$.

If $g=(x, y, \theta)$, put $r e^{i \alpha}=x+i y$. Then $x=r \cos \alpha, y=r \sin \alpha$, and the mnth entry of $T_{g}$ is

$$
\begin{gathered}
\sum^{\infty}(-i)^{m-k} J_{m-k}(N r \cos \alpha) J_{k-n}(N r \sin \alpha) e^{-i n \theta} \\
=e^{-i n \theta} J_{m-n}(N r) e^{-(m-n)(\alpha+\pi / 2)}
\end{gathered}
$$

In the light of this result we will consider $g$ as a function of $r, \alpha$ and $\theta$ rather than $x, y, \theta$. We write $g=(r, \alpha, \theta)$. Putting $g_{1}=\left(r_{1}, \alpha_{1}, \theta_{1}\right), g_{2}=\left(r_{2}, \alpha_{2}, \theta_{2}\right)$ we have $g_{1} g_{2}=\left(R, \alpha, \theta_{1}+\theta_{2}\right)$ where

$$
\begin{aligned}
& R=\left(r_{1}^{2}+r_{2}^{2}+2 r_{1} r_{2} \cos \left(\theta_{1}+\alpha_{2}-\alpha_{1}\right)\right)^{\frac{1}{2}} \text { and } \\
& e^{i \alpha}=e^{i \alpha_{1}}\left[\frac{r_{1}+r_{2} e^{i\left(\alpha_{2}-\alpha_{1}+\theta_{1}\right)}}{r_{1}+r_{2} e^{-i\left(\alpha_{2}-\alpha_{1}+\theta_{1}\right)}}\right]^{\frac{1}{2}} .
\end{aligned}
$$

Writing $\alpha_{2}-\alpha_{1}+\theta_{1}=\phi$ and substituting in (13) we have on simplification

$$
J_{m-n}(N R)\left[\frac{r_{1}+r_{2} e^{-i \phi}}{r_{1}+r_{2} e^{i \phi}}\right]^{(m-n) / 2}=\sum_{-\infty=k}^{\infty} J_{m-k}\left(N r_{1}\right) J_{k-n}\left(N r_{2}\right) e^{i \phi(n-k)}
$$

Putting $N=1, n=0$ this becomes

$$
J_{m}(R)\left[\frac{r_{1}+r_{2} e^{-i \phi}}{r_{1}+r_{2} e^{i \phi}}\right]^{m / 2}=\sum_{-\infty=k}^{\infty}(-1)^{k} J_{k+m}\left(r_{1}\right) J_{k}\left(r_{2}\right) e^{i \phi k} .
$$

This is an addition formula for Bessel functions: in fact the more familiar Graf formula may be obtained by substituting $\phi+\pi$ for $\phi$.

\section{References}

[1] F. Bingen, 'Les fonctions de Bessel d'ordre entire et les représentations du group des placements du plan', Bull. Soc. Math. de Belg. (1965), 115-152.

[2] Gelfand and Shapiro, Representations of the group of rotations (Reprinted in A. M. S. Translations Series 2, Vol. 2, 1956). 
[3] Hille and Phillips, Functional Analysis and Semigroups (A. M. S. 1957).

[4] M. A. Naimark, Normed Rings (Noordhoff 1964).

[5] E. Thoma, 'Die unitaren Darsettellungen der unwersellen Úberlagerungsgruppe der Bewegungsgruppe des $R^{2}$, Math. Ann. 134 (1958), 428-459.

[6] N. J. Vilenkin, Bessel functions and representations of the group of Euclidean movements (U. M. N. 1956 (69) Russian).

[7] N. J. Vilenkin, Special Functions and the Theory of Group Representations (A. M. S. 1968).

[8] Akhiezer and Glazman. Theory of linear operators in Hilbert Space. Volume 1. (Ungar 1966).

Monash University

Clayton, Vic. 3168 